\title{
Increased plasma homocysteine levels in shift working bus drivers
}

\author{
P J F Martins, V D'Almeida, N Vergani, A B A Perez, S Tufik
}

Occup Environ Med 2003;60:662-666

See end of article for authors' affiliations

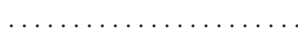

Correspondence to: Dr V D'Almeida, Rua Napoleão de Barros, 925, São Paulo, SP Brazil 04024-002;

valmeida.dped@epm.br

Accepted 6 August 2002
Background: Previous studies have indicated an association between shift work and cardiovascular disease. There is also considerable epidemiological evidence that hyperhomocysteinemia is an independent risk factor for cardiovascular disorders.

Aims: To analyse plasma homocysteine levels in shift work bus drivers, and to investigate possible relations with sleep parameters and other biochemical factors.

Methods: Blood samples were collected from 30 male shift working long-haul bus drivers in a Brazilian sample and analysed for plasma levels of homocysteine, folic acid, vitamin $B_{12}$, and serum lipids. A group of 22 daytime workers, matched for age and body mass index served as controls. The incidence of mutations in the gene coding for methylene tetrahydrofolate, an enzyme which is related to hyperhomocysteinemia, was also assessed. Polysomnographic recordings were obtained from the target group.

Results: Bus drivers showed significantly higher levels of plasma homocysteine than the control group $(18.57$ v $9.43 \mu \mathrm{M})$. Most of the other biochemical, behavioural, and molecular parameters did not differ between groups. Likewise, sleep parameters appeared to be within the normal range.

Conclusions: The significantly increased plasma homocysteine levels in long-haul bus drivers did not appear to be secondary to other biochemical or behavioural problems in this group. These results suggest that hyperhomocysteinemia may be involved in the increased incidence of cardiovascular diseases observed in shift workers.
T he number of employees engaged in shift work seems to be growing, mainly due to the demand for services around the clock in many occupations. In the USA approximately 20 million people ( 1 in 5) work non-standard schedules, such as rotating and night shifts. ${ }^{1}$ There is no agreement on a definition of shift work in the literature, but it often encompasses work outside conventional daytime hours and thereby encompasses fixed evening and night work, roster work, and ordinary three-shift work. ${ }^{2}$

Long working hours may cause irritability, physical and mental fatigue, excessive sleepiness, insomnia, and gastric ulcers, and may also result in loss of attention in the working place. $^{34}$ The situation is even worse in the case of shift work, which has been identified as an occupational risk factor. ${ }^{5}$ Shift work, in particular night work, causes disruption of biological rhythms and perturbation of family life, with negative effects on performance, health, and social wellbeing. Health problems may be manifested in the short term as sleep disorders, jet-lag syndrome, and increased proneness to accidents. In the long run, there are increased risks for gastrointestinal, psychoneurotic, and cardiovascular diseases (CVD). ${ }^{6}$

A recent review of 17 studies $^{2}$ indicates an association between shift work and coronary heart disease, shift workers showing a $40 \%$ increase in CVD risk in comparison to day workers. The follow up in the Helsinki Heart Study over a six year period detected a $30-50 \%$ increase in CVD risk in shift workers, with some variation in subgroups, in comparison with day workers. Despite certain differences in lifestyle, such as alcohol consumption, smoking, and body mass index, and despite highly significant differences in levels of systolic blood pressure, none of these factors was sufficient to explain the increased incidence of CVD among shift workers. ${ }^{7}$ Knutsson and colleagues ${ }^{8}$ compared patients with acute first time myocardial infarction with asymptomatic control subjects obtained from the same population. Myocardial infarction risk was positively associated with shift work both in men and women, an effect which could not be accounted for by factors such as job strain, age, job education level, or smoking. Peter and colleagues ${ }^{9}$ confirmed the direct effects of shift work on CVD and, in addition, verified that a stressful psychosocial work environment may act as a mediator of health-adverse effects, much like factors such as hypertension and atherogenic lipids.

While CVD risk is probably multifactorially determined, ${ }^{2}$ the literature has focused on the behaviour of shift workers and has largely neglected other possible causal connections and contributing factors. Efforts to identify such factors in this population are important not only to substantiate the association between CVD and shift work, but also to inform the elaboration of effective measures for prevention of the illness.

Currently, one of the most studied risk factors for CVD is levels of homocysteine (Hcy), an amino acid which stands at the intersection of two major metabolic pathways, remethylation to methionine and transsulphuration to cystathionine. ${ }^{10}$ Hyperhomocysteinemia is thought to promote both atherogenesis and thrombosis, although the pathophysiological mechanisms are far from completely understood. ${ }^{11}$ Nevertheless, the association between hyperhomocysteinemia and vascular disease is strongly supported by epidemiological evidence from case-control, cross sectional, and prospective cohort studies, which have identified hyperhomocysteinemia as a graded cardiovascular risk factor, independent of other

Abbreviations: AHI, apnoea/hypopnoea index; CVD, cardiovascular disease; Hcy, homocysteine; HDL, high density lipoprotein; LDL, low density lipoprotein; MTHFR, methylene tetrahydrofolate reductase; OR, odds ratio; REM, rapid eye movement; REML, REM sleep latency; SEff, sleep efficiency; SOL, sleep onset latency; SWS, slow wave sleep; TST, total sleep time; VLDL, very low density lipoprotein; WASO, wakefulness after sleep onset 
conventional risk factors. ${ }^{11-14}$ Factors that could potentially lead to hyperhomocysteinemia include some genetic defect in one of the enzymes involved in Hcy metabolism or a nutritional deficiency of one or more of the vitamins that participate in Hcy metabolism (folic acid and vitamins $\mathrm{B}_{6}$ and $\left.\mathrm{B}_{12}\right){ }^{.10}$

While Hcy is associated with CVD and the latter is very frequent in shift workers, no studies, to the best of our knowledge, have investigated levels of Hcy in shift workers. Therefore this was the main objective of this study. To this end we studied a sample of Brazilian long-haul bus drivers working shifts. Our analyses also included behavioural factors, sleep, and biochemical and molecular parameters.

\section{MATERIAL AND METHODS \\ Study subjects}

Data were obtained from 30 male professional long-distance bus drivers ranging in age between 31 and 51 years (mean age 40.6 (SD 5.77) years) and body mass index (BMI) between 18.5 and 37.0. These drivers were shift workers employed by the same interstate transportation company, who followed irregular schedules-that is, whose driving assignments to varying interstate destinations might start at different times of day or night. Subjects included in the study were equated for average duration of driving assignments, which was approximately six hours each trip, with a compulsory rest period of at least 12 hours between trips. The median time at this occupation for this sample was 37 months (range 4-106 months). Mandatory pre-employment medical examinations had been carried out, which screened for pre-existing chronic diseases, including cardiovascular disease, in addition to annual medical examinations mandated by law. They enrolled as volunteers in a sleep study at the Sleep Institute, Department of Psychobiology, Federal University of São Paulo.

Another 22 male workers equated for age (43.5 (5.14) years) and BMI served as controls. These subjects worked regular (non-shift) schedules in several occupations that were not significantly different from bus driving in socioeconomic terms. Each subject answered a yes-no questionnaire which asked whether they often engaged in physical activity, consumed alcohol, and/or smoked. All subjects gave informed written consent to participate in the study and agreed with the procedures, according to the norms of the Medical Ethics Committee of UNIFESP/EPM. The investigation conformed with the principles outlined in the Declaration of Helsinki.

\section{Blood sampling}

Fasting blood samples for biochemical analyses were collected at 8 am into vacuum tubes (Becton Dickinson). One day before sampling, all subjects underwent the following standardised procedure: they performed a work shift during the day, arrived at the sleep laboratory at $8 \mathrm{pm}$, and were directed to bed at 10 $\mathrm{pm}$. Before going to the laboratory, they had dinner at the bus company headquarters at $7 \mathrm{pm}$. After an uninterrupted night of sleep, fasting blood was collected at $8 \mathrm{am}$. They then had breakfast and answered a questionnaire. Subjects stayed awake until $10 \mathrm{pm}$ when polysomnographic recordings were carried out for eight hours. Blood samples were processed, and serum and plasma aliquots were separated and stored at $-80^{\circ} \mathrm{C}$ until biochemical analyses were carried out.

\section{Biochemical methods}

Plasma folic acid and vitamin $\mathrm{B}_{12}$ were determined by the automated chemiluminescence system (ACS:180, Bayer Corporation). Total Hcy values were determined by high performance liquid chromatography (HPLC) with fluorimetric detection and isocratic elution. ${ }^{15}$ Commercial kits (DIALAB, Diapack Liquid Reagents, Vienna, Austria) were used for spectrophotometric serum determinations of total cholesterol
Table 1 Characteristics of self reported daytime and bus driver shift workers

\begin{tabular}{lll}
\hline Variable & Daytime workers (n) & Bus drivers (n) \\
\hline Smoker (\%) & $35.00(7)$ & $37.50(9)$ \\
Alcohol consumer (\%) & $47.62(10)$ & $58.33(14)$ \\
Physically active (\%) & $33.33(7)$ & $75.00(18)$ * \\
BMl (mean \pm SD) & $25.64 \pm 3.77(22)$ & $27.30 \pm 3.61(30)$ \\
\hline
\end{tabular}

BMI, body mass index

*Significantly different from daytime workers $\left(\chi^{2}\right.$ test, $\left.p<0.05\right)$.

and its fractions (HDL, LDL, and VLDL), triglycerides, and glucose by ADVIA 16/50 (Bayer Diagnostics Corporation).

\section{Sleep recording}

All 30 drivers underwent polysomnographic assessment after an initial adaptation night to the sleep laboratory. Sleep recordings were carried out for eight hours (beginning at $2200 \pm 0.30 \mathrm{~h}$ ) using Medilog Recorders (Oxford Instruments Ltd). The following parameters were obtained, according to the criteria established by Rechtschaffen and Kales ${ }^{16}$ : sleep onset latency (SOL; time lag between the beginning of recording and the first 10 minutes of uninterrupted sleep), total sleep time (TST; sum of all sleep periods during the recording), sleep efficiency (SEff, TST/total recording time), duration of stages 1 and 2, duration of stages 3 and 4 slow wave sleep (SWS), duration of rapid eye movement sleep (REM), REM latency (REML), wakefulness after sleep onset (WASO; sum of all periods of wakefulness after sleep onset), number of arousals/hour, apnoea/hypopnoea index (AHI; number of apnoea and hypopnoea events/hour).

\section{Gene analysis}

Genomic DNA was obtained from peripheral blood samples using QIAamp DNA Mini Kit (QIAGEN Inc.). To analyse the C677T mutation in the methylene tetrahydrofolate reductase (MTHFR) gene, $50 \mathrm{ng}$ of DNA was used for polymerase chain reaction (PCR), amplification, using upstream and downstream primers flanking the respective sequences. ${ }^{17}$ The amplified and digested PCR products were analysed in $8 \%$ non-denaturing ethidium bromide stained polyacrylamide gels, and each individual was genotyped.

\section{Statistical analysis}

Living habits and biochemical parameters were analysed using non-parametric tests for comparison between groups after distribution analysis. Data for biochemical variables are expressed both as means (SD) and medians (range), since not all variables followed a normal distribution. Data for sleep parameters are presented as means (SEM) for ease of comparison to published values. Correlation analyses were performed using Spearman's rank correlation test $\left(r_{\mathrm{s}}\right)$. The association of occupation types with risk factors for CVD, folic acid, and vitamin $\mathrm{B}_{12}$, including C677T polymorphism, was evaluated using unadjusted and adjusted odds ratio (OR) computed with logistic regression analyses. For all analyses, the alpha level was established at $p \leqslant 0.05$ (two tailed) conducted with the SAS statistical system (SAS Institute, Inc., North Carolina, USA).

\section{RESULTS}

\section{Sample characteristics}

Table 1 shows the living habits of both groups. Statistical analyses revealed no significant differences between groups for most of the behavioural variables, except for a significantly higher percentage of physically active bus drivers compared to controls $\left(\chi^{2}=6.28, \mathrm{p}<0.05\right)$. 
Table 2 Biochemical parameters in shift and daytime workers

\begin{tabular}{|c|c|c|c|c|c|}
\hline \multirow[b]{2}{*}{ Variable } & \multicolumn{2}{|c|}{ Daytime workers $(n=22)$} & \multicolumn{2}{|c|}{ Shift workers $(n=30)$} & \multirow[b]{2}{*}{$\mathrm{p}$ value } \\
\hline & Mean (SD) & Median (range) & Mean (SD) & Median (range) & \\
\hline Glycaemia (mg/dl) & $92.62(13.13)$ & 89.85 (71.97-120.00) & $96.07(45.48)$ & $88.00(70.00-319.00)$ & 0.31 \\
\hline Total cholesterol (mg/dl) & $194.05(39.41)$ & 190.35 (133.33-298.00) & $179.11(29.97)$ & $179(100.00-244.00)$ & 0.25 \\
\hline Triglycerides (mg/dl) & $152.21(71.34)$ & $148.55(43.35-312.00)$ & $139.15(54.02)$ & $134.00(67.00-334.00)$ & 0.50 \\
\hline $\mathrm{HDL}$ cholesterol (mg/dl) & $49.11(13.52)$ & $49.59(30.99-78.00)$ & $39.14(11.18)$ & $36.50(19.00-74.00)$ & 0.01 \\
\hline $\mathrm{LDL}$ cholesterol (mg/dl) & $113.14(36.17)$ & 110.85 (57.99-197.00) & $112.31(31.05)$ & $108.8531 .20-144.40$ ) & 0.88 \\
\hline VLDL cholesterol (mg/dl) & $30.42(14.23)$ & $29.71(8.67-62.00)$ & $27.78(10.61)$ & $26.80(13.40-66.80)$ & 0.50 \\
\hline $\begin{array}{l}\text { Total cholesterol:HDL cholesterol } \\
\text { ratio }\end{array}$ & $4.21(1.36)$ & $3.97(2.52-7.64)$ & $4.99(1.76)$ & $4.97(2.04-9.42)$ & 0.09 \\
\hline Homocysteine $(\mu M)$ & $9.43(3.25)$ & $9.22(4.80-18.23)$ & $18.57(9.54)$ & $16.23(8.41-57.82)$ & $<0.01$ \\
\hline Vitamin $B_{12}(\mathrm{pg} / \mathrm{ml})$ & $430.20(188.79)$ & 385.75 (190.40-1069.00) & $367.76(114.26)$ & $351.70(130.70-734.10)$ & 0.26 \\
\hline Folic acid (ng/ml) & $8.46(3.91)$ & $7.80(2.7-20.00)$ & $5.08(2.23)$ & $5.00(2.4-13.60)$ & $<0.01$ \\
\hline
\end{tabular}

\begin{tabular}{lll}
$\begin{array}{l}\text { Table } 3 \\
\text { MTHFR }\end{array}$ & Presence of C677T polymorphism in the \\
\hline C677T & $\begin{array}{l}\text { Daytime workers } \\
\mathrm{n}(\%)\end{array}$ & $\begin{array}{l}\text { Shift workers } \\
\mathrm{n}(\%)\end{array}$ \\
\hline genotype & $11(50.00)$ & $19(63.33)$ \\
$\mathrm{CC}$ & $9(41.00)$ & $11(36.67)$ \\
$\mathrm{CT}$ & $2(9.00)$ & $0(0)$ \\
$\Pi \pi$ &
\end{tabular}

C677T polymorphism: CC, homozygous for the wild type; CT, heterozygous, and TT, homozygous for the mutation.

\section{Biochemical measures}

Table 2 summarises the results. Both groups had biochemical parameters within normal range, with the exception of Hcy level in bus drivers, which was $>15 \mu \mathrm{M}$ (normal range 5-15 $\mu \mathrm{M})$. Furthermore, $66.7 \%$ of bus drivers versus $9.1 \%$ of day workers had Hcy levels above $15 \mu \mathrm{M}$, which is often used to define increased risk for $\mathrm{CVD}^{18}$ (odds ratio (OR) 20.0, 95\% CI 3.88 to 103.1). There were also significant differences between daytime and shift workers in the levels of HDL cholesterol $(-20 \%, \mathrm{p}<0.05)$, Hcy $(+96 \%, \mathrm{p}<0.05)$, and folic acid $(+40 \%, \mathrm{p}<0.05)$. There were no significant correlations among Hcy levels and other biochemical parameters in bus drivers. A stepwise multiple logistic regression analysis including all factors revealed that only homocysteine presented a significant association with shift work bus drivers ( $p<0.001$; OR $1.84,95 \%$ CI 1.28 to 2.64$)$.

\section{Sleep parameters}

All of the measured sleep parameters in the bus driver sample appeared to be well within the normal range. ${ }^{16}{ }^{19}$ Means and standard errors were: sleep onset latency: 15.75 (3.56) minutes; total sleep time: 401.1 (12.06) minutes; sleep efficiency: $84.17 \%$ (2.15\%); percentage of sleep spent in stage 1: $8.82 \%(1.18 \%)$; stage 2 : $49 \%$ (1.96\%); slow wave sleep: $20.69 \%$ (1.88\%); and REM sleep: $21.11 \%$ (1.04\%); REM latency: 90.26 (9.73) minutes; wakefulness after sleep onset: $15.95 \%$ (3.54\%); number of arousals/hour: 5.58 (0.73) events/ h); apnoea/hypopnoea index: 6.10 (2.14).

Significant correlations were found between Hcy levels and sleep onset latency $\left(r_{\mathrm{s}}=-0.58\right)$, sleep efficiency $\left(r_{\mathrm{s}}=0.39\right)$, and number of apnoea episodes/hour $\left(r_{\mathrm{s}}=0.43\right)$ in the group of bus drivers.

\section{Gene analyses}

The prevalence of the heterozygous and homozygous $677 \mathrm{C} \rightarrow \mathrm{T}$ polymorphism in the MTHFR gene in the group of bus drivers was not different from the prevalence in controls $\left(\chi^{2}=1.49\right.$; $\mathrm{p}>0.05$ ) (table 3 ). There were no significant correlations between C677T mutation genotypes and the levels of Hcy, folic acid, or vitamin $B_{12}$ in shift worker or control groups.

\section{DISCUSSION}

The present study revealed increased plasma Hcy levels in 67\% of the shift workers studied, indicating an increased cardiovascular risk. These data are consistent with the higher incidence of CVD observed in other populations of shift workers. $^{26-8} 2021$

Among the hypotheses proposed to explain the augmented susceptibility of shift workers to develop CVD, Härma and Ilmarinen ${ }^{22}$ have suggested that shift work triggers the effect of other lifestyle related risk factors for coronary heart disease. The risk factors that have been studied in this population include disruption of circadian rhythms, disturbed sociotemporal patterns, disrupted social support, stress, behaviour (smoking, diet, alcohol, exercise), and biochemical changes (for example, increased levels of cholesterol and triglycerides). ${ }^{2}$

In this study the percentage of smokers was similar in the two groups (table 1). It has been suggested that for both daytime and rotating-shift workers the number of cigarettes smoked per day is associated with poor work site social support and/or lower work pace control. ${ }^{23}$ The lack of differences related to smoking in the present study is consistent with this possibility. It is quite possible therefore that increased smoking is not directly related to shift work; rather, it may be a mediating factor which cannot completely explain the increased risk for CVD among shift workers. ${ }^{24}$

In the literature there are controversial data concerning alcohol consumption among shift workers. In the Helsinki Heart Study, the number of moderate or heavy alcohol consumers was apparently lower among shift workers compared to daytime workers. On the other hand, in the Copenhagen male study there was no difference in the proportion of self reported drinkers between shift and daytime workers; however, among the drinkers, shift workers consumed slightly, but significantly more than day workers. ${ }^{24}$ In our study no differences in the proportion of alcohol consumption were found between the groups (table 1).

In general, there is no indication of marked differences in physical activity between shift and daytime workers, which is of interest given the general association between physical activity levels and cardiovascular risk. ${ }^{7824}$ In our sample, $75 \%$ of the shift workers reported regularly engaging in physical activity, as defined by Caspersen and colleagues, ${ }^{25}$ in contrast to $33 \%$ of control subjects. However, given that BMI indices did not differ between groups, the amount of physical activity reported by the shift work group may not be sufficient to produce alterations which may be translated in a lower risk for cardiovascular disease.

Bøggild and Knutsson ${ }^{2}$ observed a slight increase of cholesterol and trygliceride levels in shift workers, which is an important observation considering that these and other lipids are the best markers of atherosclerotic diseases. Peter and 
colleagues ${ }^{9}$ reported an increased total/HDL cholesterol ratio in shift workers. Contrary to what has been previously reported, the values of lipids in our bus drivers were within the normal range. Although HDL cholesterol levels were lower in bus drivers than in daytime workers, the total/HDL cholesterol ratio was similar between both groups (table 2 ).

Homocysteine has been considered an independent risk factor for cardiovascular diseases. ${ }^{118}$ No previous studies however have analysed plasma levels of this amino acid in shift workers. The shift workers in the present study had homocysteine levels well above the normal range. Elevation of plasma homocysteine levels may provide an explanation for the higher incidence of cardiovascular diseases in shift workers, a problem which has not been satisfactorily accounted for by traditional risk factors for atherosclerosis. Moderate hyperhomocysteinemia has been associated with genetic factors, chiefly polymorphisms in the gene coding for the enzyme MTHFR. ${ }^{26}$ The frequency of the particular polymorphism examined in this present study (C677T, table 3) was not different in shift workers, compared to the control group or to values in the overall Brazilian population. ${ }^{27}$ Therefore, this finding does not support the possibility that a genetic factor associated with this enzyme explains the increased levels of homocysteine detected in the bus drivers.

Another important factor associated with homocysteine levels is the availability of folic acid and vitamin $B_{12}$. Vitamin $\mathrm{B}_{12}$ status was not different between the groups and was within the normal range. In healthy subjects, however, vitamin $\mathrm{B}_{12}$ seems to play a minor role as determinant of Hcy concentration..$^{28}$ Our sample was composed of subjects with folic acid levels within the normal range, although on average, shift workers did show lower levels than daytime workers $(5.08 v 8.46 \mathrm{ng} / \mathrm{ml})$. Although the relation between folic acid and homocysteine levels is well known, there was no significant correlation between these parameters among the bus drivers. However, when we considered all the subjects (daytime and shift workers) a significant correlation emerged $\left(r_{\mathrm{s}}=-0.46\right)$, confirming the existence of a general relation between these two compounds.

The recommended amount of folic acid intake is $300 \mu \mathrm{g} / \mathrm{day}$, whereas the plasma concentration in healthy subjects is $7-8$ $n M^{28}$ The slight reduction in plasma folic acid levels in bus drivers may contribute to the increase in homocysteine levels, although folic acid levels were within expected values. The difference in folic acid levels may be related to the quality of meals taken by the shift workers. Although we have not found studies establishing an association between vitamin status and atherosclerotic diseases in shift workers, other findings have suggested altered quality and quantity of meals taken by these workers, which could affect the risk for CVD. Alternatively, it is possible that shift work does not change the diet's nutritional quality or meal frequency, ${ }^{29}$ but may instead lead to a circadian redistribution of relative energy expenditure. ${ }^{30} 31$ Redistribution of meals from the afternoon to the evening is associated with increased total and LDL cholesterol; however, when meals are taken in the morning there is a reduction in HDL cholesterol..$^{32}$ Recently, it has been observed that proteinrich meals taken in the morning can increase plasma homocysteine levels by $15-20 \%$ above fasting levels. ${ }^{18}$ Experimental studies with rats show that cholesterol and methionine supplemented diets can produce striking increases in homocysteine levels. ${ }^{33}$ It is possible, therefore, that meal redistribution to which shift working bus drivers are constantly subjected, added to the poor quality of these meals (possibly including insufficient folic acid intake), may result in a predisposition towards higher homocysteine levels. This in turn may be responsible for the high incidence of atherosclerotic diseases in shift workers. If this reasoning is correct, folic acid supplementation should be considered as a preventive measure in this population, as in the case of neural tube defects and CVD in general. ${ }^{143}$
Reduced folic acid levels combined with increased homocysteine plasma levels has been linked to heavy or chronic smoking and alcohol intake. ${ }^{28}{ }^{34}$ In the present study there were no significant differences in numbers of drinkers and smokers in the two groups. We also failed to find differences in homocysteine and folic acid levels when we separated drinkers from non-drinkers and smokers from non-smokers. Therefore, we have no evidence that lower folic acid and higher homocysteine levels in bus drivers could be attributable to smoking or alcohol intake. In summary, an important finding of this study is that Hcy was the only variable showing a significant association with this type of occupation, as shown by multiple logistic regression analyses.

On the other hand, one of the limitations of the present study was that it did not control adequately for some potentially relevant factors associated with shift work. In particular, information was not available on diet, stress assessments, and, in the case of the control group, sleep parameters. Also unavailable was information on the biochemical status of the participants before they became bus drivers shift workers. On the other hand, there is no reason to suspect that these drivers, as a group, might already have hyperhomocysteinemia before they started to work irregular schedules. Given the median length of employment ( 37 months) in their present occupation, it is unlikely that previous status would be a factor in the results obtained in this study.

The most important health complaint of shift workers is related to sleep impairments, with a higher incidence in subjects older than 40 years of age. ${ }^{41}$ The circadian resynchronisation to which shift workers are frequently subjected leads to a significant reduction in sleep time. ${ }^{35}$ There is limited knowledge on the possible relation between reduced or disrupted sleep and CVD. Sleep apnoea, for example, is a risk factor for CVD, but few studies have looked at the relation between shift work and sleep apnoea syndrome. ${ }^{236}$ Although we did find a modest correlation between the number of apnoea episodes and homocysteine levels among bus drivers, we also found that only 3.3\% (1 subject in 30) presented a pathological apnoea/hypopnoea index (AHI >10/hour), a low prevalence similar to that described by Mitler and colleagues ${ }^{36}$ for long-haul truck drivers. Overall, in the present study there was little indication of disrupted sleep patterns in bus drivers. It must be noted however that this suggestion is based on published normative data, ${ }^{19}$ since unfortunately it was not possible to obtain polysomnographic data for the control group in this study. It is also conceivable that sleep abnormalities might have emerged if the subjects had not been allowed one night of adaptation to the laboratory environment before measurements were taken. With these limitations in minds, it seems nonetheless possible to suggest that alterations in plasma Hcy levels were not secondary to sleep deficits.

\section{Conclusions}

Long-haul bus drivers working irregular shift hours were found to have increased plasma homocysteine levels, which could not be accounted for by any of the other factors examined in this study. While more investigation is required in order to explain the reason for this increase, the data suggest that hyperhomocysteinemia may be involved in the recently described increased index of cardiovascular diseases in shift workers.

\section{ACKNOWLEDGEMENTS}

The authors would like to thank Prof. Maria Tereza Cartaxo Muniz, the graduate student Eduardo Henrique Rosa Santos, and undergraduate student Allan Chiaratti de Oliveira for their input. The valuable critical comments of Prof José N Nobrega are greatly appreciated.

Financial support: Fapesp (Proc. 97/01870-4) and AFIP. Paulo JF Martins is the recipient of a fellowship from CAPES. 


\section{Authors' affiliations}

P J F Martins, V D'Almeida, S Tufik, Department of Psychobiology, Universidade Federal de São Paulo-Escola Paulista de Medicina, São Paulo, Brazil

N Vergani, A B A Perez, Department of Morphology, Universidade Federal de São Paulo-Escola Paulista de Medicina

\section{REFERENCES}

1 National Institutes of Health. Working group report on problem sleepiness (Bethesda): National Heart, Lung, and Blood Institute/National Center on Sleep Disorders Research. 1997 Available at http://www.nhlbi.nih.gov/health/prof/sleep/index.htm

2 Bøggild H, Knutsson A. Shift work, risk factors and cardiovascular disease. Scand J Work Environ Health 1999;25:85-99.

3 Florez-Lozano JA. Aspectos psicofisiológicos da fadiga. Rev Bras Saúde Ocupac 1990;29:19-23

4 Mello MT, Santana MG, Souza LM, et al. Sleep patterns and sleep-related complaints of Brazilian interstate bus drivers. Braz J Med Biol Res 2000;33:71-7.

5 Kristensen TS. Cardiovascular diseases and the work environment: a critical review of the epidemiologic literature on nonchemical factors. Scand J Work Environ Health 1989;15:165-79.

6 Costa G. Shift work and health. Med Lav 1999:90:739-51.

7 Tenkanen L, Siöblom T, Kalimo R, et al. Shift work, occupation and coronary heart disease over 6 years of follow-up in the Helsinki Heart Study. Scand J Work Environ Health 1997;23:257-65.

8 Knutsson A, Hallquist J, Reuterwall C, et al. Shiftwork and myocardial infarction: a case-control study. Occup Environ Med 1999;56:46-50.

9 Peter R, Alfredsson L, Knutsson A, et al. Does a stressful psychosocial work environment mediate the effects of shift work on cardiovascular risk factors? Scand J Work Environ Health 1999;25:376-81.

10 Selhub J. Homocysteine metabolism. Ann Rev Nutr 1999;19:217-46.

11 van der Griend R, Biesma DH, Banga J-D. Hyperhomocysteinemia as a cardiovascular risk factor: an update. Nether J Med 2000;56:1 19-30.

12 McCully KS, Wilsons RB. Homocysteine theory of arteriosclerosis. Atherosclerosis 1975;22:215-27.

13 Boushey CJ, Beresford SAA, Omenn GS, et al. A quantitative assessment of plasma homocysteine as a risk factor for vascular disease. JAMA 1995:279:1049-57.

14 Motulsky AG. Nutritional ecogenetics: homocysteine-related arteriosclerotic vascular disease, neural tube defects, and folic acid. Am J Hum Genet 1996:58:17-20.

15 Pfeiffer CM, Huff DL, Gunter EW. Rapid and accurate HPLC assay for plasma total homocysteine and cysteine in a clinical laboratory setting. Clin Chem 1999;45:290-2.

16 Rechtschaffen A, Kales A. A manual of standardized terminology techniques and scoring system for sleep stages of human subjects. Bethesda: US Department of Health, Education and Welfare, Public Health Service, 1968

17 Goyette P, Summer JS, Milos R, et al. Human methylenetetrahydrofolate reductase: isolation of $c D N A$, mapping and mutation identification. Nature Genet 1994;7:195-200.
18 Refsum $\mathrm{H}$, Ueland $\mathrm{M}$, Nygård $\mathrm{O}$, et al. Homocysteine and cardiovascular disease. Ann Rev Med 1998;49:31-62.

19 Carskadon MA, Rechstchaffen A. Monitoring and staging human sleep. In: Kryger MH, Roth T, Dement WC, eds. Principles and practice of sleep medicine, 3rd edn. Philadelphia: WB Saunders Company, 2000: 1197-215.

20 Åkerstedt T, Knutsson A, Alfredsson K, et al. Shiftwork and cardiovascular disease. Scand J Work Environ Health 1984;10:409-14 21 Morikawa Y, Nakagawa H, Miura K, et al. Relationship between shift work and onset of hypertension in a cohort of manual workers. Scand J Work Environ Health 1999:25: 100-4.

22 Härmä MI, Ilmarinen JE. Towards the 24-hour society-new approaches for aging shift workers? Scand J Work Environ 1999;25:610-15.

23 Kawakami N, Haratani T, Araki S. Job strain and arterial blood pressure, serum cholesterol, and smoking as risk factors for coronary heart disease in Japan. Int Arch Occup Environ Health 1998;71:429-32.

24 Bøggild $\mathbf{H}$, Suadicani $\mathrm{P}$, Hein $\mathrm{HO}$, et al. Shift work, social class, and ischaemic heart disease in middle aged and elderly men: a 22 year follow up in the Copenhagen male study. Occup Environ Med 1999;56:640-5.

25 Caspersen CJ, Powell KE, Christenson GM. Physical activity, exercise, and physical fitness: definitions and distinctions for health-related research. Pub Health Report 1985;100:126-31.

26 Frosst $\mathbf{P}$, Blom HJ, Milos R, et al. A candidate genetic risk factor for vascular disease: a common mutation in methylenetetrahydrofolate reductase. Nature Genet 1995;10:111-13.

27 Arruda VR, Siqueira LH, Gonçalves MS, et al. Prevalence of the mutation $\mathrm{C} 677 \rightarrow \mathrm{T}$ in the methylene tetrahydrofolate reductase gene among distinct ethnic groups in Brazil. Am J Med Genet 1998:78:332-5

28 Pietrzik K, Brönstrup A. Vitamins B12, B6 and folate as determinants of homocysteine concentration in the healthy population. Eur J Pediatr 1998; 157: S135-8.

29 Lennernäs M Hambræus L, Åkerstedt T. Nutrition and shiftwork: the use of meal classification as a new tool for qualitative/quantitative evaluation of dietary intake in shiftworkers. Ergonomics 1993;36:247-54.

30 Lennernäs $M$, Åkerstedt T, Hambræus L. Nocturnal eating and serum cholesterol of three-shift workers. Scand J Work Environ Health 1994;20:401-6.

31 Lennernäs $M$, Hambræus L, Åkerstedt T. Shift related dietary intake in day and shift workers. Appetite 1995;25:253-65.

32 Zulli A, Buxton B, Doolan L, et al. Effect of homocysteine and cholesterol in raising plasma homocysteine, cholesterol and triglyceride levels. Life Sci 1998;62:2191-4.

33 Malinow MR, Duell PB, Hess DL, et al Reduction of plasma homocyst(e)ine levels by breakfast cereal fortified with folic acid in patients with coronary heart disease. N Engl J Med 1998;388:100915

34 Christensen B, Landaas S, Stensvold I, et al. Whole blood folate, homocysteine in serum, and risk of first acute myocardial infarction, Atherosclerosis 1999:147:317-26.

35 Bliwise DL. Historical change in the report of daytime fatigue. Sleep $1996 ; 19: 462-4$

36 Mitler MM, Miller JC, Lipsitz JJ, et al. The sleep of long-haul truck drivers. N Engl J Med 1997;337:755-61. 